\title{
De novo mutations in sporadic deletional Duchenne muscular dystrophy (DMD) cases
}

\author{
Monisha Mukherjee ${ }^{1}$, L. S. Chaturvedi ${ }^{1,2}$, \\ Sandhya Srivastava ${ }^{1,3}$, R. D. Mittal ${ }^{4}$ \\ and Balraj Mittal ${ }^{1,5}$
}

\author{
${ }^{1}$ Department of Medical Genetics \\ Sanjay Gandhi Postgraduate Institute of Medical Sciences \\ Raebareli Road, Lucknow 226014, India \\ ${ }^{2}$ Present address: Henry Ford Hospital, Detroit, USA \\ ${ }^{3}$ Present address: Rajiv Gandhi Cancer Hospital, New Delhi, India \\ ${ }^{4}$ Department of Urology, Sanjay Gandhi Postgraduate \\ Institute of Medical Sciences, Raebareli Road, \\ Lucknow 226014, India \\ ${ }^{5}$ Corresponding author: Tel, 91-0522-26680004-8 (ex) 2322; \\ Fax, 91-0522 2668 017; E-mail, balraj@sgpgi.ac.in
}

Accepted March 262003

Abbreviations: CPK, creatine phosphokinase; CVS, chorionic villus sample; DMD, Duchenne muscular dystrophy; Mb, megabases; m-PCR, multiplex PCR; Q-PCR, quantitative PCR; STR, short tandem repeat

\begin{abstract}
Dinucleotide repeat polymorphism based genetic analysis is a powerful approach to gain insight into rare genetic events like germline mosaicism and de novo mutations. The loss of heterozygosity of polymorphic dinucleotide loci at "deletional hotspot" of dystrophin gene can provide direct evidence of carrier status in female relatives of affected DMD patients with overlapped exonic deletions. We have used 4 STR loci of the central deletional hotspot of the dystrophin gene for genetic analysis in sporadic unrelated DMD families. Twenty-nine mothers of sporadic deletional cases were analysed and their carrier status was determined. Eighteen of them showed heterozygosity in the deleted loci suggesting the occurrence of de novo mutations. In 9 cases, the carrier status was indeterminate while 2 showed germline mosaicism. Our observations reiterated the importance of STR analysis in determining the status of mothers of sporadic deletional DMD cases in order to provide proper genetic counselling.
\end{abstract}

Keywords: dinucleotide repeats; germline mutation; heterozygote detection; mosaicism; muscular dystrophy, Duchenne; polymorphism

\section{Introduction}

Duchenne muscular dystrophy (DMD) is an X-linked recessive lethal disorder resulting from mutation at Xp21 region. About $2 / 3$ of patients have large intragenic deletions in the dystrophin gene, which can be easily detected by multiplex PCR or Southern hybridization. However, detection of carriers in asymptomatic female relatives is tricky. Dosage analysis protocols require expensive equipment and controlled conditions, which may not be possible in most diagnostic laboratories. Linkage analyses using polymorphic dinucleotide (CA) repeat markers are widely used for the determination of carrier status. In familial cases, the mother of a patient is generally an obligate carrier and linkage methods can be effectively used provided enough loci have been utilized to rule out recombination. However, in sporadic cases, mothers might be carriers or there may be a new mutation. It is widely believed that in some $2 / 3$ of cases, mothers were carriers and remaining $1 / 3$ were due to de novo mutations. If the intragenic deletion in dystrophin gene involves one or more polymorphic CA repeat loci, then the status of the repeat loci in mothers can be used to find out whether they are carriers, non-carriers, germline mosaics or there is an occurrence of new de novo mutations in the probands. Here, we report the identification of de novo mutation in cases where probands show intragenic deletion.

\section{Materials and Methods}

\section{Selection of families}

All the patients came from Uttar Pradesh and adjoining states of North India. Families with suspected DMD patients were enrolled in the out patient door of Genetics and Neurology clinics of our Institute. Clinical diagnosis was based on physical examination, progressive muscular weakness since childhood, muscle strength, high levels of creatine phosphokinase (CPK), calf hypertrophy, positive Gower's sign, valley sign at the shoulder (Pradhan's sign) (Pradhan and Mittal, 1995) and myopathic changes on electromyelo- 
graphy. Four to five $\mathrm{ml}$ of blood from each proband and their family members were collected and DNA was isolated using standard phenol/chloroform method (Sinha et al., 1992). Twenty nine sporadic deletional DMD families were selected for our present study. The probands in all these families had intragenic deletion(s) in the central region (exons 43-52) which were detected by multiplex PCR (m-PCR) (Chamberlain et al., 1988; Beggs et al., 1990; Singh et al., 1997). There was no family history of the disease.

\section{$(\mathrm{CA})_{\mathrm{n}}$ repeat analysis}

Highly polymorphic dinucleotide $(\mathrm{CA})_{\mathrm{n}}$ loci covering the deleted region of the dystrophin gene were used for linkage analysis in the sporadic deletional families. In a few cases, there was a single exon deletion where it was made sure that the deletion in the proband extended to the corresponding intron as well. The haplotype of each STR was established by allelotyping on the basis of electrophoretic mobility of alleles. PCR was performed according to Clemens et al. (1991) with minor modifications. All polymorphic loci were amplified in duplex PCRs (STRs 44 and 49, STRs 45 and 50 ) either by $\left[\alpha-{ }^{32} \mathrm{P}\right] \mathrm{dCTP}$ incorporation or $5^{\prime}$ end labeling of primers with $\left[\gamma^{32}\right.$ P $]$ ATP. PCR products were denatured at $95^{\circ} \mathrm{C}$ for $4 \mathrm{~min}$ and electrophoresed on $8 \%$ denaturing polyacrylamide-sequencing gel $(20 \times 40 \mathrm{~cm})$ followed by autoradiography.

\section{Results}

Table 1 shows the extent of intragenic deletion in the proband of each family. The heterozygosity/homozygosity status of the STRs spanning the deleted region has been highlighted, on the basis of which the carrier status in the respective mothers was determined. Out of 29 families with sporadic cases of DMD, in 18 cases (F1-F18) there was heterozygosity at one or more deleted loci that suggested the occurrence of de novo mutation in the probands. The mothers of such probands were ascertained as noncarriers. In 2 cases (F19 and F20), the mothers were found to be germline mosaics because two off-springs with the disease and one normal son showed the inheritance of haplotype 'b' from the mother (Table 2). F7 and F21 were included in the present study since the probands showed a single exon deletion ( $\triangle 45$ and $\triangle 51$ respectively), which extended to the corresponding introns (Table 1). Lastly, there were 9 mo-

Table 1. Status of mothers of sporadic DMD patients showing deletion.

\begin{tabular}{|c|c|c|c|c|c|c|}
\hline Family No. & Deletion in proband & STR 44 & STR 45 & STR 49 & STR 50 & Status of mother \\
\hline 1. & $\Delta \mathrm{E} 48-51$ & I & I & I & I & de novo mutation \\
\hline 2. & $\Delta E 45-50$ & I & I & I & I & de novo mutation \\
\hline 3. & $\Delta \mathrm{E} 49-50$ & $\mathrm{NI}$ & $\mathrm{NI}$ & & $\mathrm{NI}$ & de novo mutation \\
\hline 4. & $\Delta \mathrm{E} 48-52$ & $\mathrm{NI}$ & $\mathrm{NI}$ & I & $\mathrm{NI}$ & de novo mutation \\
\hline 5. & $\Delta E 45-48$ & $\mathrm{NI}$ & I & $\mathrm{NI}$ & I & de novo mutation \\
\hline 6. & $\triangle 45-52$ & I & I & I & $\mathrm{NI}$ & de novo mutation \\
\hline 7. & $\Delta \mathrm{E} 45$ & I & I & I & I & de novo mutation \\
\hline 8. & $\Delta \mathrm{E} 45-50$ & I & I & I & I & de novo mutation \\
\hline 9. & $\triangle 47-52$ & i & $\mathrm{NI}$ & i & $\mathrm{NI}$ & de novo mutation \\
\hline 10. & $\triangle 44-51$ & I & $\mathrm{NI}$ & $\mathrm{NI}$ & $\mathrm{NI}$ & de novo mutation \\
\hline 11. & $\triangle 45-51$ & I & I & I & I & de novo mutation \\
\hline 12. & $\triangle 45-51$ & $\mathrm{NI}$ & $\mathrm{NI}$ & $\mathrm{NI}$ & i & de novo mutation \\
\hline 13. & $\triangle 48-51$ & $\mathrm{NI}$ & $\mathrm{NI}$ & I & $\mathrm{NI}$ & de novo mutation \\
\hline 14. & $\triangle 46-52$ & $\mathrm{NI}$ & $\mathrm{NI}$ & i & I & de novo mutation \\
\hline 15. & $\triangle 48-50$ & $\mathrm{NI}$ & $\mathrm{NI}$ & i & $\mathrm{NI}$ & de novo mutation \\
\hline 16. & $\Delta 47-51$ & I & $\mathrm{NI}$ & $\mathrm{NI}$ & I & de novo mutation \\
\hline 17. & $\triangle 45-48$ & I & I & I & I & de novo mutation \\
\hline 18. & $\triangle 44-51$ & $\mathrm{NI}$ & $\mathrm{NI}$ & I & $\mathrm{NI}$ & de novo mutation \\
\hline 19. & $\Delta \mathrm{E} 47-52$ & I & I & l & I & Germline mosaicism \\
\hline 20. & $\Delta \mathrm{E} 48$ & i & $\mathrm{NI}$ & I & $\mathrm{NI}$ & Germline mosaicism \\
\hline 21. & $\Delta \mathrm{E} 51$ & i & I & $\mathrm{NI}$ & $\mathrm{NI}$ & Carrier? \\
\hline 22. & $\Delta \mathrm{E} 47-50$ & $\mathrm{NI}$ & I & $\mathrm{NI}$ & $\mathrm{NI}$ & Carrier? \\
\hline 23. & $\Delta \mathrm{E} 45-47$ & $\mathrm{NI}$ & $\mathrm{NI}$ & I & $\mathrm{NI}$ & Carrier? \\
\hline 24. & $\Delta \mathrm{E} 47-50$ & $\mathrm{NI}$ & 1 & NI & I & Carrier? \\
\hline 25. & $\triangle 47-52$ & $\mathrm{NI}$ & $\mathrm{NI}$ & $\mathrm{NI}$ & $\mathrm{NI}$ & Carrier? \\
\hline 26. & $\Delta 50-52$ & I & $\mathrm{NI}$ & I & $\mathrm{NI}$ & Carrier? \\
\hline 27. & $\triangle 45-48$ & i & NI & i & $\mathrm{NI}$ & Carrier? \\
\hline 28. & $\triangle 47-51$ & I & $\mathrm{NI}$ & $\mathrm{NI}$ & $\mathrm{NI}$ & Carrier? \\
\hline 29. & $\triangle 47-51$ & I & $\mathrm{NI}$ & $\mathrm{NI}$ & $\mathrm{NI}$ & Carrier? \\
\hline
\end{tabular}

I, informative; NI, non-informative 
Table 2. Summary of de novo mutations and germline mosaicism in sporadic deletion DMD cases.

\begin{tabular}{cccc}
$\begin{array}{c}\text { Total No. of } \\
\text { mothers }\end{array}$ & $\begin{array}{c}\text { De novo } \\
\text { cases }\end{array}$ & $\begin{array}{c}\text { Germline } \\
\text { mosaics }\end{array}$ & $\begin{array}{c}\text { Indeterminate } \\
\text { cases }\end{array}$ \\
\hline 29 & 18 & 2 & 9 \\
\hline
\end{tabular}

thers (F21-F29), whose carrier status could not be determined due to the homozygosity or loss of heterozygosity of the STRs in the desired region (Tables 1 and 2).

Figure 1 shows the family F2 where the proband (II-3) had an intragenic deletion of exons 45-49. The deletion of STR 45 in the proband is shown in lane 1 in Figure 1B. However, the mother (I-2) had two alleles (heterozygous, 3/1) for the same locus (Figures $1 \mathrm{~A}, \mathrm{~B}$ ) and also transmitted the 'b' haplotype to her normal son who had no intragenic deletion (Figure 1A). The proband was therefore assumed to have a de novo mutation.

$\mathbf{A}$ $\mathrm{F} 2(\Delta 45-49)$

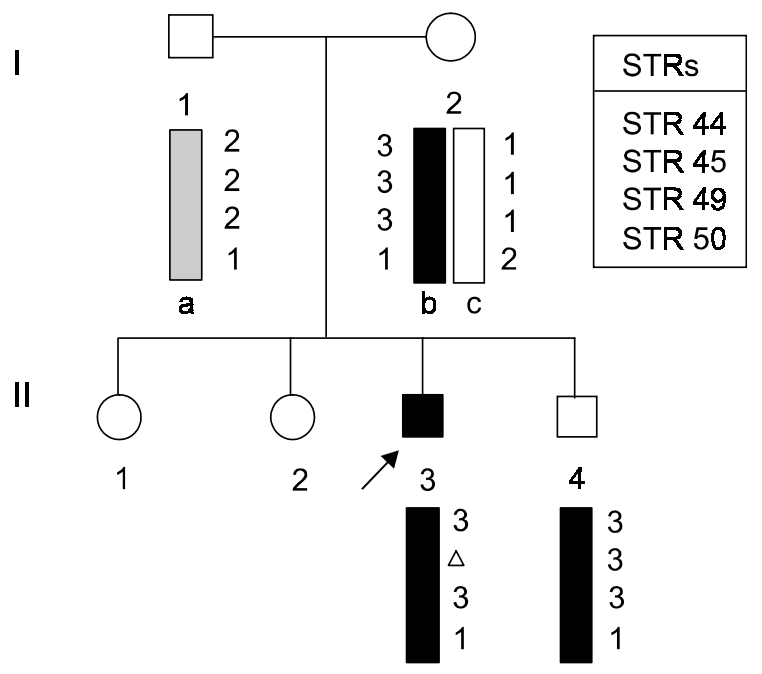

B

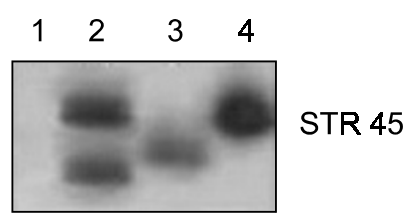

Figure 1. (A) Pedigree of family F2 showing the haplotypes of father ' $a$ ', mother ' $b$ ', ' $c$ ' and male children. The intragenic deletion on 'b' haplotype is observed only in the proband (II-3). (B) Pattern of STR 45. Lane 1, proband (II-3) where STR is deleted; lane 2, mother (I-2) showing heterozygosity; lane 3, father (I-1) and lane 4, normal brother (II-4).
A

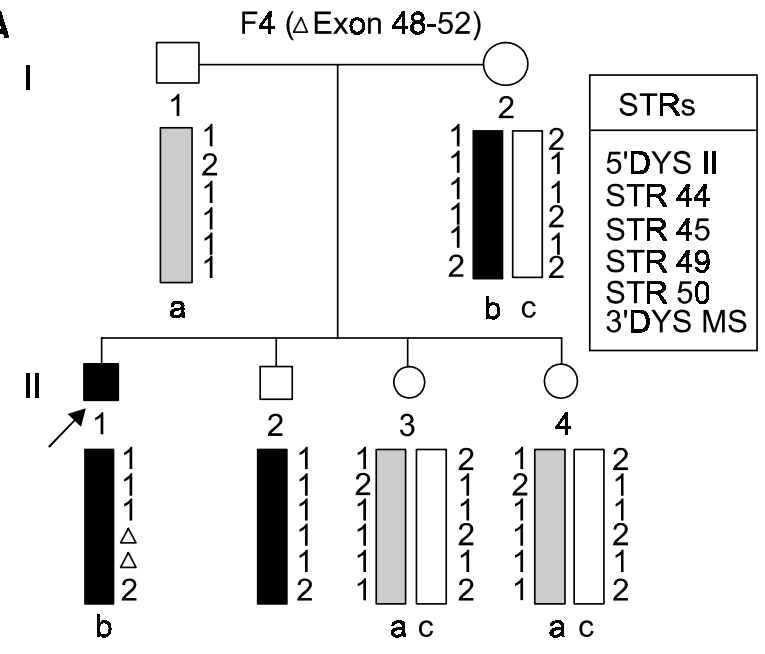

B

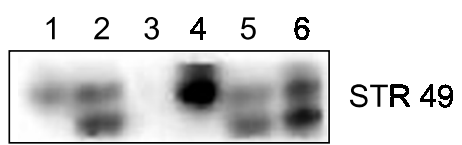

Figure 2. (A) Pedigree of family F4 showing the haplotypes of father ' $a$ ', mother ' $b$ ', ' $c$ ' and children. The haplotype ' $b$ ' which is inherited by the proband (II-1)shows the deletion of STRs 49 and 50. (B) Pattern of STR 49. Lane 1, father (I-1), lane 2, mother (I-2) showing heterozygosity; lane 3, proband (II-1) where STR is deleted; and lane 4, normal male (II-2); lanes 5 and 6 , sisters (II-3, II-4).

Figrue 2 shows a family F4 where the proband (II-1) showed a deletion of exons 48-52 which included STRs 49 and 50 . The mother (I-2) was heterozygous for at least one of the deleted STRs (STR 49) (Figure 2B), so the occurrence of de novo mutation in the proband was confirmed. The female sibs (II-3 and

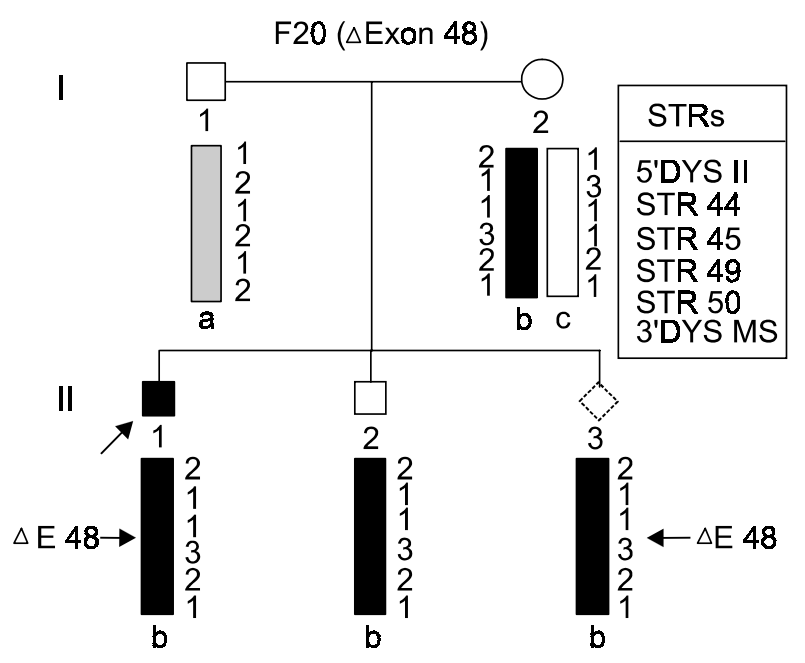

Figure 3. Pedigree of family F20 showing 2 affected males, [proband (II-1) and CVS (II-3)] and a normal male child (II-2) all sharing 'b' haplotype of the mother. 


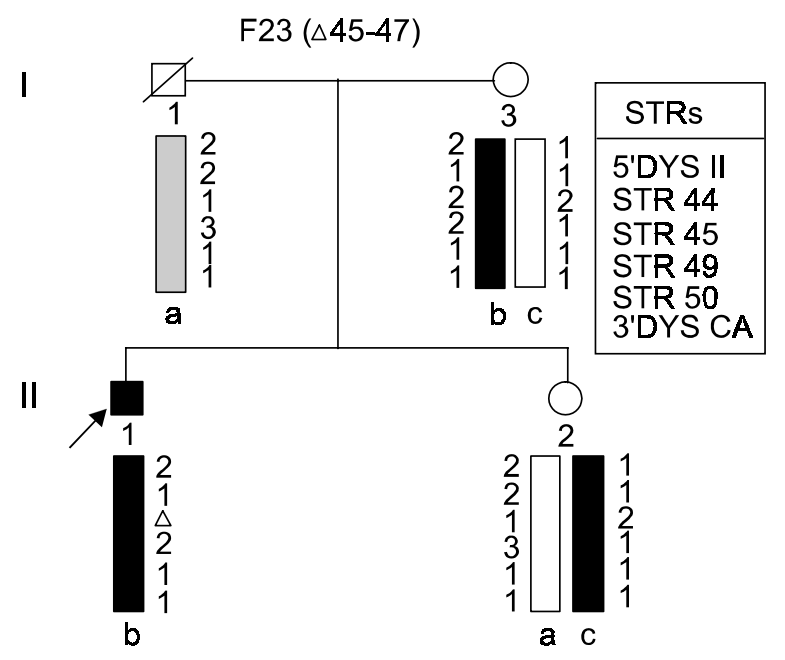

Figure 4. Pedigree of family F23 showing that the mother $(\mathrm{I}-3)$ is homozygous $(2 / 2)$ at the STR 45 locus which is deleted in the proband (II-1).

II-4) were not carriers because they had inherited the normal maternal haplotype (Figure 2A).

Figure 3 shows a family F20, where a deletion of a single exon 48 was detected in the proband (II-1) and CVS (II-3). The male foetus (CVS), proband and normal male child (II-2) all shared the same maternal haplotype suggesting that the mother (I-2) is a germline mosaic.

Figure 4 shows a family F23 where the mother (I-2) was non-informative (homozygous) at the STR 45 locus, which was within the deleted region (exons 45-47). In such cases the carrier status of the mothers was indeterminate.

\section{Discussion}

In case of sporadic cases, STR analysis of mothers/ probands is important to determine the possible carrier status of the mother. Genetic counseling to female relatives of patients with DMD is difficult due to the enormous size of the gene $(2.4 \mathrm{Mb})$, mutational heterogeneity (deletions, duplications and point mutations), high rate of intragenic recombinations and high frequency of new mutations (Koenig et al., 1987; Gillard et al., 1989; Hu et al., 1990, Abbs and Bobrow 1992; Prior et al., 1995; Sinha et al., 1996; Alcantara et al., 1999). Quantitative PCR have been used to provide the carrier status but still these methods have not been well standardized thereby increasing the utility of STR markers for linkage analysis. Highly polymorphic $(C A)_{n}$ loci are very useful tools for carrier analysis because the probability of recombination between STR markers and deletional hotspot would be quite low. Therefore, only four STR loci of the central region identified will suffice for $\sim 80 \%$ of the deletional DMD families in India due to the high prevalence of deletions at the central hotspot region. For carrier determination in familial cases, these STRs are able to ascertain the carrier risk in majority of female relatives (95\% confidence level) (Chaturvedi et al., 2001).

However, in sporadic cases, the carrier status in mothers will depend on two situations. Firstly, if the mother is hemizygous (homozygous) for the deleted STR locus (loci), she may or may not be considered a carrier. Secondly, if the mother is heterozygous for the deleted STR locus (loci), she can either be a germline mosaic (more than two affected sibs with identical mutations) with higher carrier risk; or she may not be a carrier at all because of the occurrence of de novo mutations in the proband. Eighteen sporadic mothers, F1-F18 (Tables 1 and 2) having only one affected male child in each case was found to be heterozygous for deleted STR locus (loci). The STR analysis confirmed that the affected sons of respective families were due to the occurrence of de novo deletions at the central hotspot region because the deleted STR locus (loci) had been detected only in the affected sons. Therefore, mothers of these families would be considered as non-carriers. However, in particularly small families the mothers may be mosaics. To completely rule out the risk due to germline mosaicism, it will be advisable to carry out prenatal diagnosis even in sporadic de novo cases.

Two mothers of sporadic cases, F19 and F20 were determined as germline mosaics. In these mothers the deletional mutations probably occurred at some stage after differentiation of progenitor germ cells. Therefore, upto $50 \%$ of mothers' germ cells might have been at risk of having the mutated copy of the gene. These females have a higher risk of transmitting the disease to their offsprings (Wood and McGillivray, 1988; Bakker et al., 1989).

Germline mosaicism and de novo mutations probably result from new mutations. In case the mutation event takes place at a very early stage in differentiation, a larger proportion of the derived germ cells may carry the mutation and the mutant maternal allele can be transmitted to more than one offspring. However, if the mutation takes place at a later stage, fewer cells are likely to manifest it as a de novo mutation.

In the present study, we have observed new mutations including germline mosaicism in 20 out of 29 sporadic mothers. These findings are consistent with our earlier observations based on Q-PCR analysis (Sinha et al., 1996).

So far, STR analysis has mainly been used for carrier and prenatal diagnosis in non-deletional DMD families as an indirect tool for linkage study (Arenas 
et al., 1996; Shiroshita and Katayama 1997; Baranzini et al., 1998). Our results, however, clearly depict the relevance of $(C A)_{n}$ loci for carrier analysis and prenatal diagnosis in deletional DMD families with no previous history of the disease.

\section{Acknowledgement}

Two of us (MM and SS) are thankful to CSIR, New Delhi for providing Research Fellowships.

\section{References}

Abbs S, Bobrow M. Analysis of quantitative PCR for the diagnosis of deletion and duplication carriers in the dystrophin gene. J Med Genet 1992;29:191-6

Arenas D, Coral R, Cisneros B, Penaloza L, Salamanca F, Kofamn S, Mercado R, Mendez J, Martinez C, Montanez C. Carrier detection in Duchenne and Becker muscular dystrophy using dinucleotide repeat polymorphisms. A study in Mexican families. Arch Med Res 1996;27:151-6

Bakker E, Veenema H, Den Dunnen JT, Van Broeckhoven C, Grootscholten PM, Bonten EJ, Van Ommen GJB, Pearson $\mathrm{PL}$. Germinal mosaicism increases the recurrence risk for 'new' Duchenne muscular dystrophy mutations. J Med Genet 1989;26:553-9

Baranzini SE, Giliberto F, Dalamon V, Barreiro C, GarciaErro M, Grippo J, Szijan I. Carrier detection in Duchenne and Becker muscular dystrophy Argentine families. Clin Genet 1998;54:503-11

Beggs AH, Koenig M, Boyce FM, Kunkel LM. Detection of $98 \%$ of DMD/BMD gene deletions by polymerase chain reaction. Hum Genet 1990;86:45-6

Chamberlain JS, Gibbs RA, Ranier JE, Nguyen PN, Caskey C. Deletion screening of the Duchenne muscular dystrophy locus via multiplex DNA amplification. Nucl Acid Res 1988; 16:11141-56

Chaturvedi LS, Srivastava S, Mukherjee M, Mittal RD, Phadke SR, Pradhan S, Mittal B. Carrier detection in non-deletional Duchenne/Becker muscular dystrophy families using polymorphic dinucleotide (CA) repeat loci of dystrophin gene. Ind J Med Res 2001;113:19-25
Clemens PR, Fenwick RG, Chamberlain JS, Gibbs RA, De Andrade M, Chakraborty R, Caskey CT. Carrier detection and prenatal diagnosis in Duchenne and Becker muscular dystrophy families, using dinucleotide repeat polymorphisms. Am J Hum Genet 1991;49:951-60

Gillard EF, Chamberlain JS, Murphy EG, Duff CL, Burghes AHM, Thompson MW, Sutherland J, Oss Y, Bodburg SE, Klamut HJ, Ray PN, Worton RG. Molecular and phenotypic analysis of patients with deletion within the deletion-rich region of the Duchenne muscular dystrophy (DMD) gene. Am J Hum Genet 1989;45:507-20

Hu X, Ray PN, Murphy E, Thompson MW, Worton RG. Duplicational mutation at the Duchenne muscular dystrophy locus: its frequency, distribution, origin and phenotype/genotype correlation. Am J Hum Genet 1990;46:682-95

Koenig M, Hoffman EP, Bertelson CJ, Monaco AP, Feener CA, Kunkel LM. Complete cloning of the Duchenne muscular dystrophy (DMD) CDNA and preliminary genomic organization of the DMD gene in normal and affected individuals. Cell 1987;50:509-17

Pradhan S, Mittal B. Infraspinatus muscle hypertrophy and wasting of axillary folds as the important sign in Duchenne muscular dystrophy. Clin Neurol Neurosurg 1995;97:134-8

Prior TW, Bartolo C, Pearl DK, Papp AC, Snyder PJ, Sedra MS, Burghes AHM, Mendell JR. Spectrum of small mutations in the dystrophin coding region. Am J Hum Genet 1995;57: 22-33

Shiroshita Y, Katayama S. Prenatal diagnosis of Duchenne muscular dystrophy in the Japanese population by flourescent CA repeat polymorphisms analysis. J Obstet Gynaecol Res 1997;23:453-61

Singh V, Sinha S, Mishra S, Chaturvedi LS, Pradhan S, Mittal RD, Mittal B. Proportion and pattern of dystrophin gene deletions in north Indian Duchenne and Becker muscular dystrophy patients. Hum Genet 1997;99:206-8

Sinha S, Pradhan S, Mittal RD, Mittal B. Detection of gene deletion in patients of Duchenne muscular dystrophy/Becker muscular dystrophy using polymerase chain reaction. Indian J Med Res 1992;96:297-301

Sinha S, Mishra S, Singh V, Mittal RD, Mittal B. High frequency of new mutations in north Indian Duchenne/Becker muscular dystrophy patients. Clin Genet 1996;50:327-31

Wood S, McGillivray BC. Germinal mosaicism in Duchenne muscular dystrophy. Hum Genet 1988;78:282-84 